\title{
What Is the Role of Resistance Exercise in Improving the Cardiometabolic Health of Adolescents with Obesity?
}

\author{
SoJung Lee ${ }^{1, *}$, YoonMyung Kim², Jennifer L. Kuk ${ }^{3}$ \\ 'Division of Sports Medicine, Graduate School of Physical Education, Kyung Hee University, Yongin; ${ }^{2}$ University College, Yonsei University International Campus, \\ Incheon, Korea; ${ }^{3}$ School of Kinesiology and Health Science, York University, Toronto, ON, Canada
}

\begin{abstract}
Traditionally, individuals with obesity have been encouraged to participate in aerobic exercise for long-term weight management and improved obesity-related health outcomes. Recently, resistance exercise has become a popular mode of exercise among youth with obesity. However, to date, the literature is mixed as to whether resistance exercise training alone improves body weight, fat free mass, body composition, cardiovascular risk factors, or atherogenic lipoprotein profiles. The limited research in this area suggests potential sex differences in response to resistance training in youth. The literature is more consistent in demonstrating improvements in muscular fitness and insulin resistance independent of caloric restriction and weight loss. Although major health organizations recommend combining aerobic and resistance training, little research has examined the effects of their combination versus their individual effects, thus it is unclear whether their combination is associated with benefits that extend beyond those of either exercise modality alone. The purpose of this review is to examine the effects of resistance exercise on body composition and the health risk factors associated with cardiovascular disease and type 2 diabetes in youth with obesity.
\end{abstract}

Key words: Childhood obesity, Resistance exercise, Abdominal adiposity, Insulin resistance, Cardiovascular disease risk

\author{
Received May 19, 2019 \\ Reviewed June 5, 2019 \\ Accepted June 7, 2019 \\ *Corresponding author \\ SoJung Lee \\ (1) \\ https://orcid.org/0000-0002-6634-6800 \\ Division of Sports Medicine, Graduate \\ School of Physical Education, Kyung Hee \\ University, 1732 Deogyeong-daero, \\ Giheung-gu, Yongin 17104, Korea \\ Tel: +82-31-201-2751 \\ Fax: +82-31-204-8117 \\ E-mail: sojung.lee@khu.ac.kr
}

\section{INTRODUCTION}

According to the World Health Organization ${ }^{1}$, the number of children and adolescents with obesity has increased from 11 million to 124 million during the past four decades. Although variations in the prevalence of childhood obesity exist across countries ${ }^{1}$, it is now apparent that childhood obesity is a major public health concern worldwide.

It is well documented that children and adolescents with obesity are more likely than normal-weight children and adolescents to have obesity in adulthood ${ }^{2,3}$, with a heightened risk of developing hypertension, dyslipidemia, insulin resistance, type 2 diabetes, and non-alcoholic fatty liver disease. ${ }^{48}$ Of particular health concern is an increase in waist circumference in children and adolescents over time ${ }^{9}$ since enlarged waist circumferences indicate increased visceral adiposity and is associated with numerous cardiometabolic risk factors independent of body mass index (BMI). ${ }^{10-12}$

Previous studies have demonstrated that cardiorespiratory fitness (CRF) protects against obesity-related comorbid conditions in children ${ }^{13-16}$ Emerging evidence also suggests that muscular strength, another main component of health-related physical fitness, is an important factor in the prevention of chronic health conditions. ${ }^{17}$ In adults, muscular strength is inversely associated with all-cause-, cancer-, and cardiovascular disease (CVD)-related mortality inde-

Copyright (C) 2019 Korean Society for the Study of Obesity

(a) This is an Open Access article distributed under the terms of the Creative Commons Attribution Non-Commercial License (http://creativecommons.org/licenses/by-nc/4.o/) which permits unrestricted non-commercial use, distribution, and reproduction in any medium, provided the original work is properly cited. 
pendent of BMI and CRF. ${ }^{18,19}$ In children and adolescents, evidence suggests that muscular strength measured by a 1-repetition maximum (1-RM) or hand grip strength test is associated with lower insulin resistance and inflammatory biomarkers independent of CRF. ${ }^{20,21}$ These findings support the current public physical activity guidelines that children and adolescents (age 6-17 years) should engage in muscle strengthening physical activity at least 3 days a week, in addition to regular aerobic physical activity. ${ }^{22}$

The purpose of this review is to examine the effects of resistance exercise training on abdominal fat, insulin resistance, and CVD risk factors in children and adolescents with obesity. Given that major health authorities (e.g., Public Health Agency of Canada, U.S. Department of Health and Human Services, World Health Organization) recommend a combination of resistance and aerobic exercise training, we also explore the effects of combining resistance and aerobic exercise on cardiometabolic risk factors in children and adolescents.

\section{EFFECTS OF RESISTANCE EXERCISE ON BODY COMPOSITION}

Lifestyle interventions to increase physical activity and promote a healthy diet have been the first-line approach for treating youth with obesity. Because childhood obesity is a strong predictor of adult morbidity and early mortality ${ }^{23}$, early intervention is essential to prevent and reverse obesity-related risk factors in youth. Traditionally, adults with obesity have been encouraged to participate in aerobic exercise for long-term weight management. During the past decade, resistance exercise has also become a popular mode of exercise in youth with obesity, and some studies have demonstrated that engaging in resistance exercise improves muscular strength and lean body mass or skeletal muscle mass ${ }^{2426}$ and provides psychological benefits. ${ }^{27,28}$

Resistance exercise, both alone and combined with dietary interventions (e.g., reducing caloric restriction or sugar intake), has been examined for its effects on total and abdominal obesity in children and adolescents (Table 1). As shown in Table 1, most studies have used short-duration resistance exercise regimens (6-16 weeks); one study lasted 36 weeks. ${ }^{29}$ Furthermore, most studies have used exercise durations of 40-60 minutes per session, 3 day/wk, and the vast majority of studies reported no significant weight loss following resistance training. For example, Shaibi et al. ${ }^{24}$ examined the effects of a 16-week progressive resistance training program without caloric restriction ( 2 day/wk, 60 minutes per session, 10 single and multi-joint resistance exercises) on total fat measured using dualenergy X-ray absorptiometry (DXA) in a small sample of overweight Latino boys (BMI > 85th percentile). They observed no significant changes in body weight or total fat in the resistance exercise group, although significant increases in muscular strength (bench press and leg press 1-RM) and total lean body mass were found in the resistance exercise group compared with controls. Subsequently, the same research group ${ }^{30}$ conducted a 16-week randomized controlled trial to compare the effects of resistance exercise training ( 2 day/wk, 60 minutes per session, whole-body resistance exercise) combined with a carbohydrate nutrition program ( $\leq 10 \%$ of total daily calorie intake from added sugar and consuming at least $14 \mathrm{~g} / 1,000 \mathrm{kcal}$ of dietary fiber/day) versus the carbohydrate nutrition program alone on changes in body composition and metabolic markers in 54 overweight Latino boys and girls (age 14-18 years). Although the resistance exercise combined with carbohydrate nutrition group showed significantly improved muscular strength (1-RM bench press) compared with the nutrition program alone and the control group, there were no significant resistance exercise training effects on changes in body weight, total fat mass, or fat free mass (FFM) in a mixed group of boys and girls.

To our knowledge, four randomized controlled trials $\mathrm{s}^{26,31-33}$ have examined changes in abdominal obesity (measured using waist circumference) in response to resistance exercise versus non-exercising controls, and three randomized controlled trials ${ }^{26,32,33}$ have examined the effect of resistance versus aerobic exercise on visceral adiposity (Table 1). Of those, only one ${ }^{26}$ demonstrated a significant reduction in waist circumference and visceral fat following resistance exercise training. Suh et al. ${ }^{33}$ conducted a 12-week study of caloric restriction ( $>1,200 \mathrm{kcal} /$ day to prevent malnutrition, limiting dietary fat intake and snacks) combined with either resistance exercise (3 day/wk, 60 minutes per session) or aerobic exercise (3 day/wk, 40 minutes per session) on total and visceral fat and metabolic markers in 30 overweight Korean boys and girls (BMI > 85th percentile; mean age, 13.1 years). Despite an intensive individualized diet education program ( 2 day/wk for 12 weeks), significant increases 


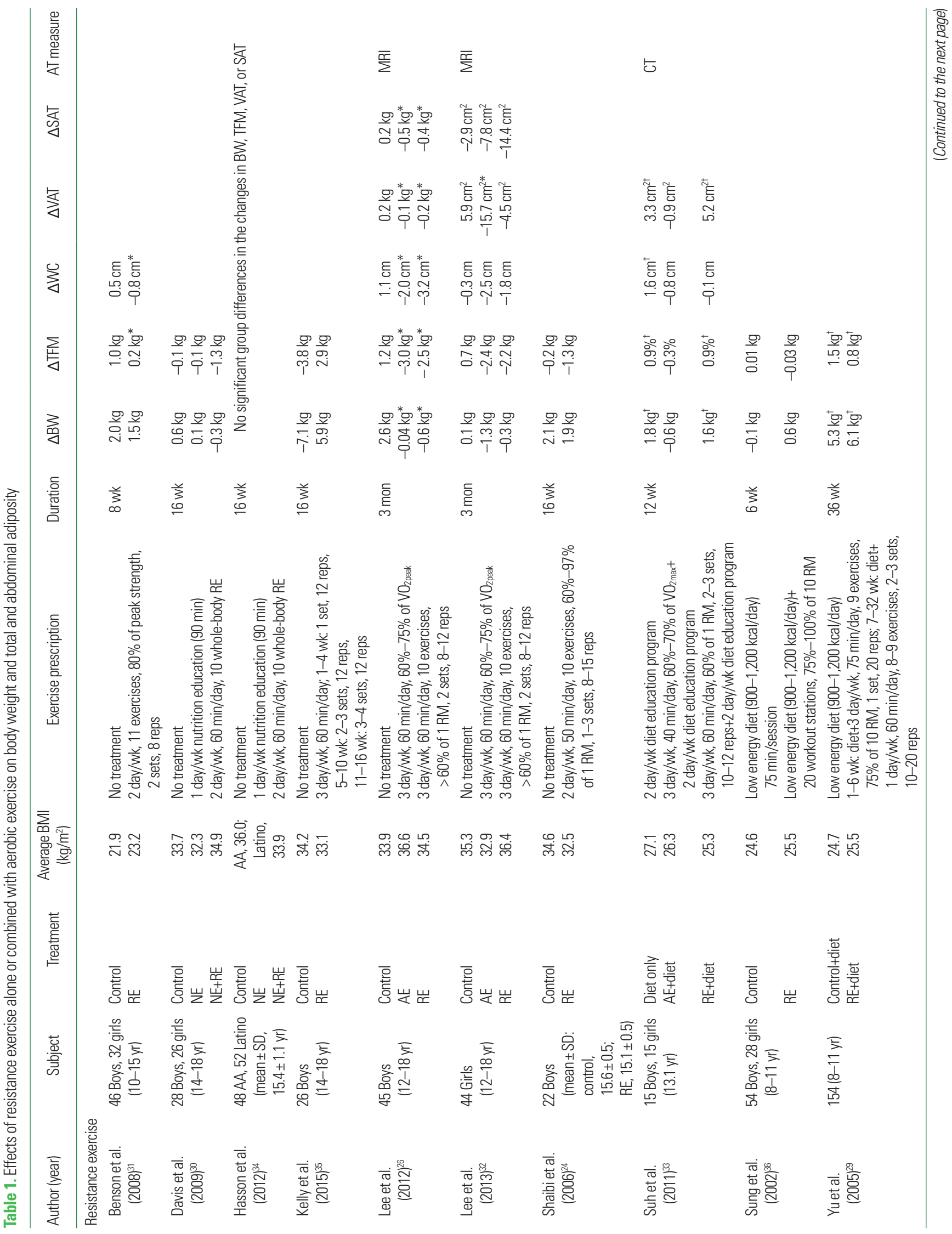




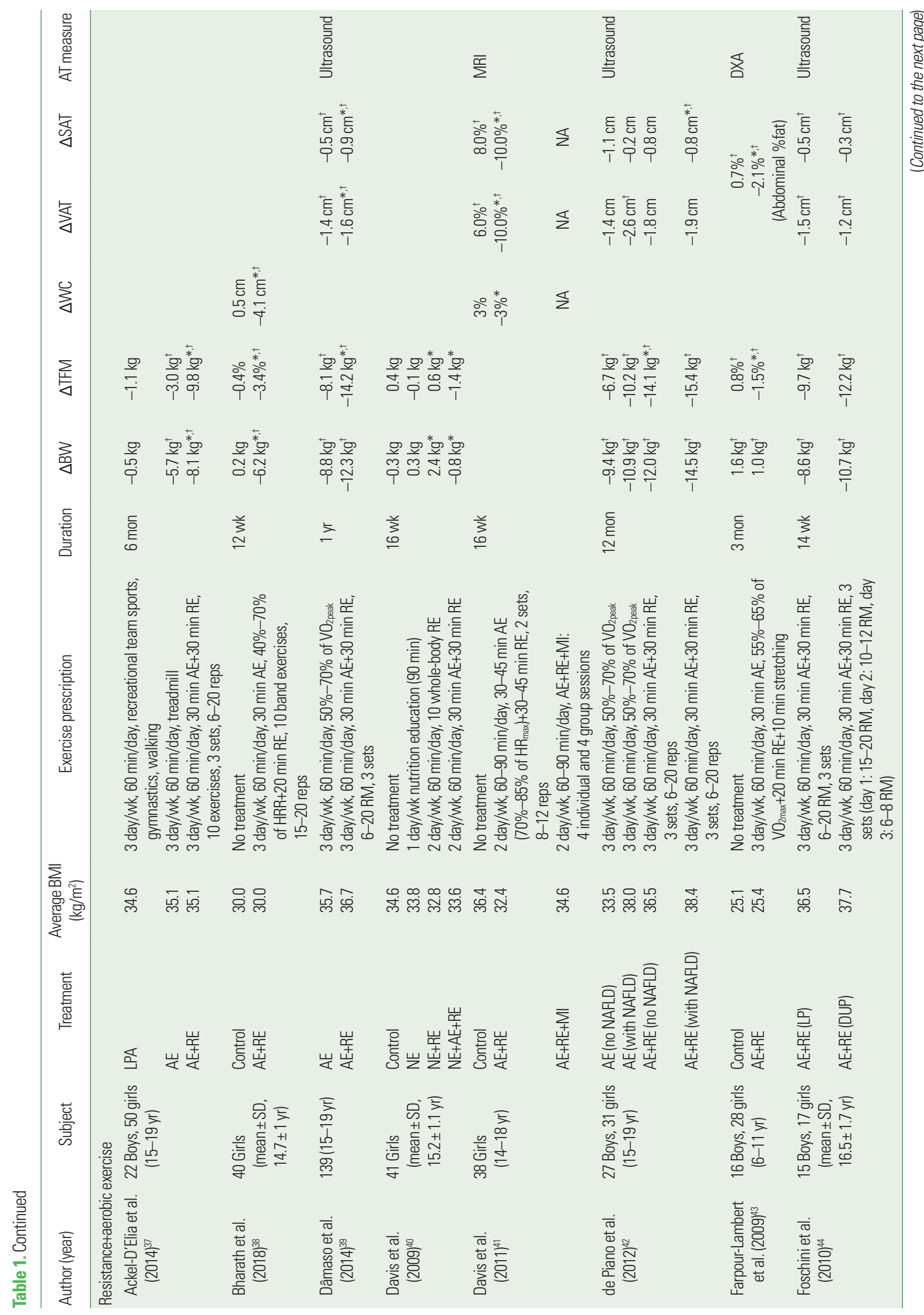




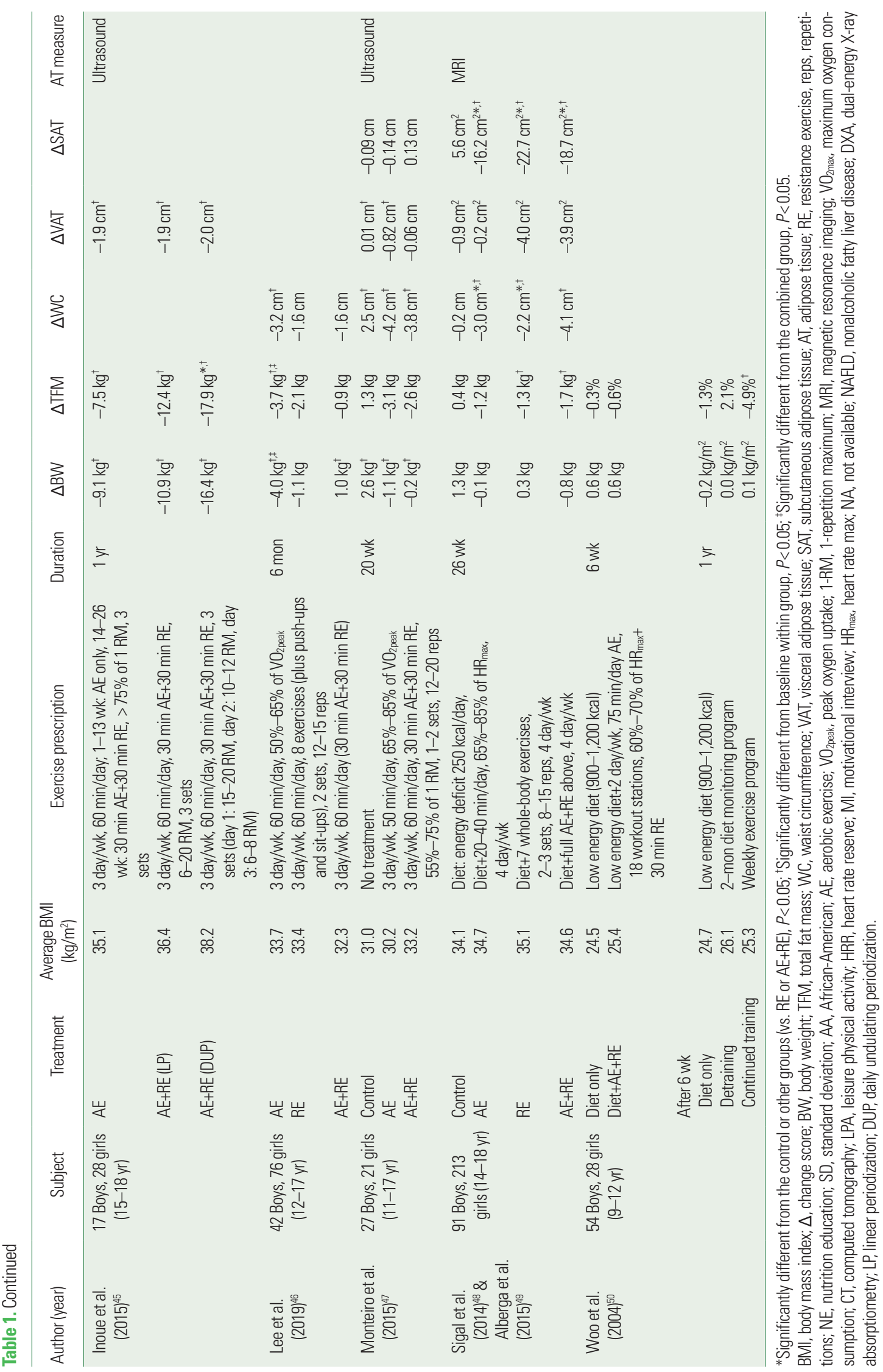


in body weight, BMI, \% body fat, and visceral fat were observed in the diet only and diet plus resistance exercise groups, but not in the diet plus aerobic exercise group. Thus, it appears that resistance exercise could be inferior to aerobic exercise at reducing visceral fat in overweight Asian adolescents.

Lee et al. ${ }^{26,32}$ conducted two randomized controlled trials to examine the effects of 3 months of resistance exercise versus aerobic exercise (no caloric restriction) on total fat and regional body fat distribution using the gold standard, whole-body magnetic resonance imaging (MRI), in adolescent boys ${ }^{26}$ and girls ${ }^{32}$ with obesity (BMI > 95th percentile, age 12-18 years). In those studies ${ }^{26,32}$, both boys and girls participated in identical aerobic and resistance exercise regimens; those who were randomized to the resistance exercise group performed one to two sets with 8-12 repetitions of eight whole-body progressive resistance exercises until volitional fatigue for 3 day/wk at 60 minutes per session plus a single set of push-ups and sit-ups, and those who were randomized to the aerobic exercise group performed moderate intensity $(60 \%-75 \%$ of peak oxygen uptake $\left[\mathrm{VO}_{2 \text { peak }}\right]$ ) aerobic exercise using treadmills and ellipticals for 3 day/wk at 60 minutes per session. Following the interventions, no significant weight loss was observed in any exercise group in either boys or girls. However, compared with the non-exercising controls, significant reductions in \% body fat (resistance, $-2.5 \%$ vs. aerobic, $-2.6 \%$ ), waist circumference (resistance, $-3.2 \mathrm{~cm}$ vs. aerobic, $-2.0 \mathrm{~cm}$ ), visceral fat (resistance, $-0.2 \mathrm{~kg}$ vs. aerobic, $-0.1 \mathrm{~kg}$ ), abdominal subcutaneous fat (resistance, $-0.4 \mathrm{~kg}$, vs. aerobic, $-0.5 \mathrm{~kg}$ ), and liver fat (resistance, $-2.0 \%$ vs. aerobic, $-1.9 \%$ ) were observed in response to both aerobic and resistance exercise training in boys, whereas in girls, reductions in visceral and liver fat were observed in response to only aerobic exercise. Furthermore, unlike the significant increases in skeletal muscle mass that occurred in response to resistance exercise in boys, adolescent girls did not have increases in skeletal muscle mass after 3 months of resistance exercise training. Given the similar exercise training regimens, MRI methodology, and high exercise attendance rates (boys, 99\% and girls, 97\%), we are uncertain about the causes of the sex differences in response to resistance exercise training. It is possible that increased androgen levels in adolescent boys allowed them to have greater hypertrophy in response to resistance exercise.

To date, the effects of resistance exercise on body composition have been inconsistent between studies, and it is currently uncertain whether engaging in resistance training alone is associated with reductions in abdominal fat in youth with obesity. The inconsistency could result from the different body composition methodologies used between studies, which varied from simple field methods (e.g., bioelectrical impedance analysis) to sophisticated imaging modalities (e.g., DXA, MRI, computed tomography [CT]). In the pediatric literature, only four studies ${ }^{26,32-34}$ out of ten ${ }^{24,26,29-36}$ randomized trials examining the effects of resistance exercise on abdominal fat used imaging modalities (e.g., MRI and CT). Among them, three ${ }^{32-34}$ of the four ${ }^{26,32-34}$ studies reported that resistance exercise did not significantly reduce abdominal fat, and one ${ }^{26}$ reported that it did reduce abdominal fat. Furthermore, most previous stud-

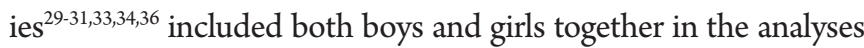
and did not examine the influence of sex on changes in study outcomes. Interestingly, the one study ${ }^{26}$ to report reductions in visceral fat with resistance training included only boys. Thus, there could be sex differences in the body composition changes associated with resistance exercise. Clearly, further randomized controlled trials with large sample sizes are required to examine the influence of sex.

\section{EFFECTS OF RESISTANCE EXERCISE ON INSULIN SENSITIVITY AND GLUCOSE TOLERANCE}

Insulin resistance has been proposed as the underlying mechanism for the development of metabolic dysfunction and type 2 diabetes in youth with obesity. ${ }^{51,52}$ Insulin-resistant children and adolescents are more likely to have a cluster of metabolic abnormalities than their insulin-sensitive counterparts. ${ }^{53}$ Although aerobic exercise has traditionally been recommended for children and adolescents with obesity, age-appropriate resistance exercise could improve musculoskeletal strength, cardiometabolic profiles, motor skills, and psychosocial well-being in youth. ${ }^{54}$

We are currently aware of eight randomized controlled trials $s^{24,26,30-35}$ that examined the effects of resistance exercise training (alone or combined with dietary modification) on insulin sensitivity and glucose tolerance in youth with overweight or obesity (Table 2). Of those eight trials, three studies ${ }^{24,26,35}$ were limited to adolescent boys. Although no studies reported significant weight loss, three of 
Table 2. Effects of resistance exercise alone or combined with aerobic exercise on insulin sensitivity and glucose tolerance

\begin{tabular}{|c|c|c|c|c|c|c|c|}
\hline Author (year) & Subject & Treatment & $\begin{array}{c}\text { Average BMl } \\
\left(\mathrm{kg} / \mathrm{m}^{2}\right)\end{array}$ & Exercise prescription & Duration & $\Delta$ Insulin resistance & Measure \\
\hline \multicolumn{8}{|c|}{ Resistance exercise } \\
\hline $\begin{array}{l}\text { Benson et al. } \\
(2008)^{31}\end{array}$ & $\begin{array}{l}46 \text { Boys, } 32 \text { girls } \\
(10-15 \mathrm{yr})\end{array}$ & $\begin{array}{l}\text { Control } \\
\text { RE }\end{array}$ & $\begin{array}{l}21.9 \\
23.2\end{array}$ & $\begin{array}{l}\text { No treatment } \\
2 \text { day/wk, } 11 \text { exercises, } 80 \% \text { of peak } \\
\text { strength, } 2 \text { sets, } 8 \text { reps }\end{array}$ & $8 w k$ & $\begin{array}{l}0.2 \\
0.1\end{array}$ & HOMA-IR \\
\hline $\begin{array}{l}\text { Davis et al. } \\
(2009)^{30}\end{array}$ & $\begin{array}{l}28 \text { Boys, } 26 \text { girls } \\
\text { (14-18 yr) }\end{array}$ & $\begin{array}{l}\text { Control } \\
\mathrm{NE} \\
\mathrm{NE}+\mathrm{RE}\end{array}$ & $\begin{array}{l}33.7 \\
32.3 \\
34.9\end{array}$ & $\begin{array}{l}\text { No treatment } \\
1 \text { day/wk nutrition education ( } 90 \mathrm{~min}) \\
2 \text { day/wk, } 60 \text { min/day, } 10 \text { whole-body RE }\end{array}$ & $16 w k$ & $\begin{array}{l}0.1 \times 10^{-4} \mathrm{~min}^{-1} / \mu \mathrm{U} / \mathrm{mL} \\
0.2 \times 10^{-4} \mathrm{~min}^{-1} / \mu \mathrm{U} / \mathrm{mL} \\
0.0 \times 10^{-4} \mathrm{~min}^{-1} / \mu \mathrm{U} / \mathrm{mL}\end{array}$ & FSIVGT \\
\hline $\begin{array}{l}\text { Hasson et al. } \\
\qquad(2012)^{34}\end{array}$ & $\begin{array}{l}48 \text { AA, } 52 \text { Latino } \\
\text { (mean } \pm \text { SD } \\
15.4 \pm 1.1 \text { yr) }\end{array}$ & $\begin{array}{l}\text { Control } \\
\mathrm{NE} \\
\mathrm{NE}+\mathrm{RE}\end{array}$ & $\begin{array}{c}\text { AA, 36.0; } \\
\text { Latino, } 33.9\end{array}$ & $\begin{array}{l}\text { No treatment } \\
1 \text { day/wk nutrition education (90 min) } \\
2 \text { day/wk, } 60 \text { min/day, } 10 \text { whole-body RE }\end{array}$ & $16 w k$ & $\begin{array}{c}\text { AA: }-0.4 \times 10^{-4} \mathrm{~min}^{-1} / \mu \mathrm{U} / \mathrm{mL} \\
\text { Latino: }-0.4 \times 10^{-4} \mathrm{~min}^{-1} / \mu \mathrm{U} / \mathrm{mL} \\
\text { AA: } 0.1 \times 10^{-4} \mathrm{~min}^{-1} / \mu \mathrm{U} / \mathrm{m}^{*} \\
\text { Latino: } 0.3 \times 10^{-4} \mathrm{~min}^{-1} / \mu \mathrm{U} / \mathrm{mL}^{*} \\
\text { AA: } 0.0 \times 10^{-4} \mathrm{~min}^{-1} / \mu \mathrm{U} / \mathrm{mL} \\
\text { Latino: }-0.2 \times 10^{-4} \mathrm{~min}^{-1} / \mu \mathrm{U} / \mathrm{mL}\end{array}$ & FSIVGTT \\
\hline $\begin{array}{l}\text { Kelly et al. } \\
(2015)^{35}\end{array}$ & 26 Boys (14-18 yr) & $\begin{array}{l}\text { Control } \\
\text { RE }\end{array}$ & $\begin{array}{l}34.2 \\
33.1\end{array}$ & $\begin{array}{l}\text { No treatment } \\
3 \text { day/wk, } 60 \text { min/day; } 1-4 \text { wk: } 1 \text { set } \\
12 \text { reps, } 5-10 \text { wk: } 2-3 \text { sets, } 12 \text { reps, } \\
11-16 \text { wk: } 3-4 \text { sets, } 12 \text { reps }\end{array}$ & $16 w k$ & $\begin{array}{c}0.4 \times 10^{-4} \mathrm{~min}^{-1} / \mu \mathrm{U} / \mathrm{mL} \\
-0.6 \times 10^{-4} \mathrm{~min}^{-1} / \mu \mathrm{U} / \mathrm{mL}\end{array}$ & FSIVGTT \\
\hline $\begin{array}{l}\text { Lee et al. } \\
\qquad(2012)^{26}\end{array}$ & 45 Boys (12-18 yr) & $\begin{array}{l}\text { Control } \\
\mathrm{AE} \\
\mathrm{RE}\end{array}$ & $\begin{array}{l}33.9 \\
36.6 \\
34.5\end{array}$ & $\begin{array}{l}\text { No treatment } \\
3 \text { day/wk, } 60 \mathrm{~min} / \text { day, } 60 \%-75 \% \text { of } \mathrm{VO}_{2 \text { peak }} \\
3 \text { day/wk, } 60 \mathrm{~min} / \text { day, } 10 \text { exercises, } \\
>60 \% \text { of } 1 \text { RM, } 2 \text { sets, 8-12 reps }\end{array}$ & 3 mon & $\begin{array}{c}-0.1 \mathrm{mg} / \mathrm{kg} / \mathrm{min} / \mu \mathrm{U} / \mathrm{mL} \\
0.4 \mathrm{mg} / \mathrm{kg} / \mathrm{min} / \mu \mathrm{U} / \mathrm{mL} \\
0.8 \mathrm{mg} / \mathrm{kg} / \mathrm{min} / \mu \mathrm{U} / \mathrm{mL}^{*}\end{array}$ & $\begin{array}{l}\text { Euglycemic } \\
\text { clamp }\end{array}$ \\
\hline $\begin{array}{l}\text { Lee et al. } \\
\qquad(2013)^{32}\end{array}$ & 44 Girls (12-18 yr) & $\begin{array}{l}\text { Control } \\
\mathrm{AE} \\
\mathrm{RE}\end{array}$ & $\begin{array}{l}35.3 \\
32.9 \\
36.4\end{array}$ & $\begin{array}{l}\text { No treatment } \\
3 \text { day/wk, } 60 \mathrm{~min} / \text { day, } 60 \%-75 \% \text { of } \\
\mathrm{VO}_{2 \text { peak }} \\
3 \text { day/wk, } 60 \mathrm{~min} / \text { day, } 10 \text { exercises, } \\
>60 \% \text { of } 1 \mathrm{RM}, 2 \text { sets, 8-12 reps }\end{array}$ & 3 mon & $\begin{array}{l}-0.5 \mathrm{mg} / \mathrm{kg} / \mathrm{min} / \mu \mathrm{U} / \mathrm{mL}^{*} \\
0.9 \mathrm{mg} / \mathrm{kg} / \mathrm{min} / \mu \mathrm{U} / \mathrm{mL}^{*} \\
0.03 \mathrm{mg} / \mathrm{kg} / \mathrm{min} / \mu \mathrm{U} / \mathrm{mL}\end{array}$ & $\begin{array}{l}\text { Euglycemic } \\
\text { clamp }\end{array}$ \\
\hline $\begin{array}{l}\text { Shaibi et al. } \\
(2006)^{24}\end{array}$ & $\begin{array}{l}22 \text { Boys (mean } \pm \text { SD: } \\
\text { control, } 15.6 \pm 0.5 \\
\text { RE, } 15.1 \pm 0.5 \text { ) }\end{array}$ & $\begin{array}{l}\text { Control } \\
\mathrm{RE}\end{array}$ & $\begin{array}{l}34.6 \\
32.5\end{array}$ & $\begin{array}{l}\text { No treatment } \\
2 \text { day/wk, } 50 \text { min/day, } 10 \text { exercises, } \\
60 \%-97 \% \text { of } 1 \text { RM, 1-3 sets, 8-15 reps }\end{array}$ & $16 w k$ & $\begin{array}{c}0.1 \times 10^{-4} \mathrm{~min}^{-1} / \mu \mathrm{U} / \mathrm{mL} \\
0.9 \times 10^{-4} \mathrm{~min}^{-1} / \mu \mathrm{U} / \mathrm{mL}^{*,+}\end{array}$ & FSIVGTT \\
\hline \multirow[t]{2}{*}{$\begin{array}{l}\text { Suh et al. } \\
\qquad(2011)^{33}\end{array}$} & $\begin{array}{l}15 \text { Boys, } 15 \text { girls } \\
\text { (13.1 yr) }\end{array}$ & $\begin{array}{l}\text { Diet only } \\
\text { AE+diet }\end{array}$ & $\begin{array}{l}27.1 \\
26.3\end{array}$ & $\begin{array}{l}2 \text { day/wk diet education program } \\
3 \text { day/wk, } 40 \mathrm{~min} / \text { day, } 60 \%-70 \% \text { of } \\
\mathrm{VO}_{2 \max }+2 \text { day } / \mathrm{wk} \text { diet education } \\
\text { program }\end{array}$ & $12 \mathrm{wk}$ & $\begin{array}{l}318.8 \mu \mathrm{U} / \mathrm{mL} \text { in insulin AUC } \\
-3,007.9 \mu \mathrm{U} / \mathrm{mL} \text { in insulin AUC }\end{array}$ & OGT \\
\hline & & RE+diet & 25.3 & $\begin{array}{l}3 \text { day/wk, } 60 \mathrm{~min} / \text { day, } 60 \% \text { of } 1 \mathrm{RM} \text {, } \\
2-3 \text { sets, } 10-12 \text { reps }+2 \text { day/wk diet } \\
\text { education program }\end{array}$ & & $-1,646.4 \mu \mathrm{U} / \mathrm{mL}$ in insulin AUC & \\
\hline \multicolumn{8}{|c|}{ Resistance+aerobic exercise } \\
\hline $\begin{array}{l}\text { Ackel-D'Elia } \\
\quad \text { et al. } \\
(2014)^{37}\end{array}$ & $\begin{array}{l}22 \text { Boys, } 50 \text { girls } \\
(15-19 \mathrm{yr})\end{array}$ & $\begin{array}{l}\text { LPA } \\
\text { AE } \\
\text { AE+RE }\end{array}$ & $\begin{array}{l}34.6 \\
35.1 \\
35.1\end{array}$ & $\begin{array}{l}3 \text { day/wk, } 60 \text { min/day, recreational team } \\
\text { sports, gymnastics, walking } \\
3 \text { day/wk, } 60 \text { min/day, treadmill } \\
3 \text { day/wk, } 60 \text { min/day, } 30 \text { min } A E+30 \text { min } \\
\text { RE, } 10 \text { exercises, } 3 \text { sets, } 6-20 \text { reps }\end{array}$ & 6 mon & $\begin{array}{l}-0.3 \\
-0.3 \\
-0.8\end{array}$ & HOMA-IR \\
\hline $\begin{array}{l}\text { Bharath et al. } \\
\qquad(2018)^{38}\end{array}$ & $\begin{array}{l}40 \text { Girls (mean } \pm \text { SD, } \\
14.7 \pm 1 \text { yr) }\end{array}$ & $\begin{array}{l}\text { Control } \\
\mathrm{AE}+\mathrm{RE}\end{array}$ & $\begin{array}{l}30.0 \\
30.0\end{array}$ & $\begin{array}{l}\text { No treatment } \\
3 \text { day/wk, } 60 \mathrm{~min} / \text { day, } 30 \mathrm{~min} \mathrm{AE}, \\
40 \%-70 \% \text { of HRR+20 min RE, } \\
10 \text { band exercises, } 15-20 \text { reps }\end{array}$ & $12 \mathrm{wk}$ & $\begin{array}{r}0.1 \\
-4.8\end{array}$ & HOMA-IR \\
\hline $\begin{array}{l}\text { Dâmaso et al. } \\
(2014)^{39}\end{array}$ & 139 (15-19 yr) & $\begin{array}{l}\mathrm{AE} \\
\mathrm{AE}+\mathrm{RE}\end{array}$ & $\begin{array}{l}35.7 \\
36.7\end{array}$ & $\begin{array}{l}3 \text { day/wk, } 60 \text { min/day, } 50 \%-70 \% \text { of } \\
\mathrm{VO}_{2 \text { peak }} \\
3 \text { day/wk, } 60 \mathrm{~min} / \text { day, } 30 \mathrm{~min} \mathrm{AE}+30 \mathrm{~min} \\
\mathrm{RE}, 6-20 \mathrm{RM}, 3 \text { sets }\end{array}$ & $1 \mathrm{yr}$ & $\begin{array}{l}-0.2^{\dagger} \\
-0.2^{\dagger}\end{array}$ & QUICKI \\
\hline $\begin{array}{l}\text { Davis et al. } \\
\qquad(2009)^{40}\end{array}$ & $\begin{array}{l}41 \text { Girls (mean } \pm \text { SD, } \\
15.2 \pm 1.1 \mathrm{yr} \text { ) }\end{array}$ & $\begin{array}{l}\text { Control } \\
\mathrm{NE} \\
\mathrm{NE}+\mathrm{RE} \\
\mathrm{NE}+\mathrm{AE}+\mathrm{RE}\end{array}$ & $\begin{array}{l}34.6 \\
33.8 \\
32.8 \\
33.6\end{array}$ & $\begin{array}{l}\text { No treatment } \\
1 \text { day/wk nutrition education ( } 90 \mathrm{~min} \text { ) } \\
2 \text { day/wk, } 60 \mathrm{~min} / \text { day, } 10 \text { whole-body RE } \\
2 \text { day/wk, } 60 \mathrm{~min} / \text { day, } 30 \mathrm{~min} \mathrm{AE}+30 \mathrm{~min} \\
\text { RE }\end{array}$ & 16 wk & $\begin{array}{c}-0.03 \times 10^{-4} \mathrm{~min}^{-1} / \mu \mathrm{U} / \mathrm{mL} \\
0.3 \times 10^{-4} \mathrm{~min}^{-1} / \mu \mathrm{U} / \mathrm{mL} \\
-0.4 \times 10^{-4} \mathrm{~min}^{-1} / \mu \mathrm{U} / \mathrm{mL} \\
0.1 \times 10^{-4} \mathrm{~min}^{-1} / \mu \mathrm{U} / \mathrm{mL}\end{array}$ & FSIVGTT \\
\hline
\end{tabular}


Table 2. Continued

\begin{tabular}{|c|c|c|c|c|c|c|c|}
\hline Author (year) & Subject & Treatment & $\begin{array}{c}\text { Average BMl } \\
\left(\mathrm{kg} / \mathrm{m}^{2}\right)\end{array}$ & Exercise prescription & Duration & $\Delta$ Insulin resistance & Measure \\
\hline \multirow{3}{*}{$\begin{array}{l}\text { Davis et al. } \\
(2011)^{41}\end{array}$} & \multirow[t]{3}{*}{38 Girls (14-18 yr) } & Control & 36.4 & No treatment & \multirow[t]{3}{*}{$16 w k$} & $-4.0 \%^{\dagger}$ & \multirow[t]{3}{*}{ HOMA-IR } \\
\hline & & $\mathrm{AE}+\mathrm{RE}$ & 32.4 & $\begin{array}{l}2 \text { day/wk, } 60-90 \mathrm{~min} / \text { day, } 30-45 \mathrm{~min} A \mathrm{AE} \\
\left(70 \%-85 \% \text { of } \mathrm{HR}_{\max }\right)+30-45 \mathrm{~min} \mathrm{RE} \\
2 \text { sets, 8-12 reps }\end{array}$ & & $-21.0 \% *,+$ & \\
\hline & & $\mathrm{AE}+\mathrm{RE}+\mathrm{Ml}$ & 34.6 & $\begin{array}{l}2 \text { day/wk, } 60-90 \mathrm{~min} / \text { day, } 4 \text { individual and } \\
4 \text { group sessions }\end{array}$ & & NA & \\
\hline \multirow{4}{*}{$\begin{array}{l}\text { de Piano et al. } \\
(2012)^{42}\end{array}$} & \multirow{4}{*}{$\begin{array}{l}27 \text { Boys, } 31 \text { girls } \\
\text { (15-19 yr) }\end{array}$} & AE (no NAFLD) & 33.5 & 3 day/wk, $60 \mathrm{~min} /$ day, $50 \%-70 \%$ of $\mathrm{VO}_{2 \text { peak }}$ & \multirow[t]{4}{*}{12 mon } & -0.1 & \multirow[t]{4}{*}{ HOMA-IR } \\
\hline & & AE (with NAFLD) & 38.0 & 3 day/wk, $60 \mathrm{~min} /$ day, $50 \%-70 \%$ of $\mathrm{VO}_{2 \text { peak }}$ & & -1.6 & \\
\hline & & $\begin{array}{l}\mathrm{AE}+\mathrm{RE} \\
\text { (no NAFLD) }\end{array}$ & 36.5 & $\begin{array}{l}3 \text { day/wk, } 60 \text { min/day, } 30 \text { min AE+30 min } \\
\text { RE, } 3 \text { sets, 6-20 reps }\end{array}$ & & $-1.4^{*}$ & \\
\hline & & $\begin{array}{l}\text { AE+RE (with } \\
\text { NAFLD) }\end{array}$ & 38.4 & $\begin{array}{l}3 \text { day/wk, } 60 \text { min/day, } 30 \text { min AE } 30 \text { min } \\
\text { RE, } 3 \text { sets, 6-20 reps }\end{array}$ & & $-2.1^{*}$ & \\
\hline \multirow{2}{*}{$\begin{array}{l}\text { Farpour- } \\
\text { Lambert } \\
\text { et al. } \\
(2009)^{43}\end{array}$} & \multirow{2}{*}{$\begin{array}{l}16 \text { Boys, } 28 \text { girls } \\
\text { (6-11 yr) }\end{array}$} & Control & 25.1 & No treatment & \multirow[t]{2}{*}{3 mon } & $0.62^{\dagger}$ & \multirow[t]{2}{*}{ HOMA-IR } \\
\hline & & $A E+R E$ & 25.4 & $\begin{array}{l}3 \text { day/wk, } 60 \mathrm{~min} / \text { day, } 30 \mathrm{~min} \mathrm{AE} \\
55 \%-65 \% \text { of } \mathrm{VO}_{2 \max }+20 \mathrm{~min} \mathrm{RE}+10 \mathrm{~min} \\
\text { stretching }\end{array}$ & & $0.99^{\dagger}$ & \\
\hline \multirow[t]{2}{*}{$\begin{array}{l}\text { Foschini et al. } \\
(2010)^{44}\end{array}$} & \multirow{2}{*}{$\begin{array}{c}15 \text { Boys, } 17 \text { girls } \\
\text { (mean } \pm \text { SD } \\
16.5 \pm 1.7 \mathrm{yr})\end{array}$} & $A E+R E(L P)$ & 36.5 & $\begin{array}{l}3 \text { day/wk, } 60 \text { min/day, } 30 \text { min AE+30 min } \\
\text { RE, } 6-20 \text { RM, } 3 \text { sets }\end{array}$ & \multirow[t]{2}{*}{14 wk } & -0.3 & \multirow[t]{2}{*}{ HOMA-IR } \\
\hline & & $\mathrm{AE}+\mathrm{RE}$ (DUP) & 37.7 & $\begin{array}{l}3 \text { day/wk, } 60 \text { min/day, } 30 \text { min } A E+30 \text { min } \\
\text { RE, } 3 \text { sets (day 1: } 15-20 \mathrm{RM} \text {, day 2: } \\
\text { 10-12 RM, day 3: 6-8 RM) }\end{array}$ & & $-1.2^{\dagger}$ & \\
\hline \multirow[t]{3}{*}{$\begin{array}{l}\text { Inoue et al. } \\
\qquad(2015)^{45}\end{array}$} & \multirow[t]{3}{*}{$\begin{array}{l}17 \text { Boys, } 28 \text { girls } \\
\text { (15-18 yr) }\end{array}$} & $\mathrm{AE}$ & 35.1 & $\begin{array}{l}3 \text { day/wk, } 60 \text { min/day, } 1-13 \text { wk: AE only, } \\
\text { 14-26 wk: } 30 \text { min AE+30 min RE, > 75\% } \\
\text { of } 1 \text { RM, } 3 \text { sets }\end{array}$ & \multirow[t]{3}{*}{$1 \mathrm{yr}$} & $-8.2 \%$ & \multirow[t]{3}{*}{ HOMA-IR } \\
\hline & & $A E+R E(L P)$ & 36.4 & $\begin{array}{l}3 \text { day/wk, } 60 \mathrm{~min} / \text { day, } 30 \mathrm{~min} \mathrm{AE}+30 \mathrm{~min} \\
\mathrm{RE}, 6-20 \mathrm{RM}, 3 \text { sets }\end{array}$ & & $-48.9 \% \%^{*, t}$ & \\
\hline & & $\mathrm{AE}+\mathrm{RE}$ (DUP) & 38.2 & $\begin{array}{l}3 \text { day/wk, } 60 \text { min/day, } 30 \text { min } A E+30 \text { min } \\
\text { RE, } 3 \text { sets (day 1: } 15-20 \mathrm{RM} \text {, day 2: } \\
\text { 10-12 RM, day 3: 6-8 RM) }\end{array}$ & & $-46.2 \% *,+$ & \\
\hline \multirow{3}{*}{$\begin{array}{l}\text { Lee et al. } \\
(2019)^{46}\end{array}$} & \multirow{3}{*}{$\begin{array}{l}42 \text { Boys, } 76 \text { girls } \\
\text { (12-17 yr) }\end{array}$} & $\mathrm{AE}$ & 33.7 & 3 day/wk, $60 \mathrm{~min} /$ day, $50 \%-65 \%$ of $\mathrm{VO}_{2 \text { peak }}$ & \multirow[t]{3}{*}{6 mon } & $1.7 \mathrm{mg} / \mathrm{kg} / \mathrm{min}^{\mathrm{t} . \neq}$ & \multirow{3}{*}{$\begin{array}{l}\text { Euglycemic } \\
\text { clamp }\end{array}$} \\
\hline & & RE & 33.4 & $\begin{array}{l}3 \text { day/wk, } 60 \text { min/day, } 8 \text { exercises, } 2 \text { sets, } \\
\text { 12-15 reps }\end{array}$ & & $0.7 \mathrm{mg} / \mathrm{kg} / \mathrm{min}^{\dagger}$ & \\
\hline & & $\mathrm{AE}+\mathrm{RE}$ & 32.3 & $\begin{array}{l}3 \text { day/wk, } 60 \mathrm{~min} / \text { day ( } 30 \mathrm{~min} \mathrm{AE}+30 \mathrm{~min} \\
\text { RE) }\end{array}$ & & $1.2 \mathrm{mg} / \mathrm{kg} / \mathrm{min}^{\dagger}$ & \\
\hline
\end{tabular}

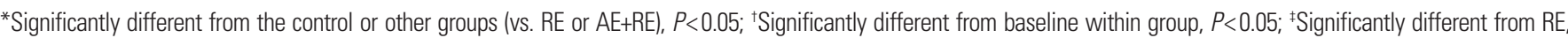
$P<0.05$.

$B M I$, body mass index; $\triangle$, change score; RE, resistance exercise, reps, repetitions; HOMA-IR, homeostatic model assessment for insulin resistance; NE, nutrition education; FSIVGTT, frequently sampled intravenous glucose tolerance test; $\mathrm{AA}$, African-American; SD, standard deviation; $\mathrm{AE}$, aerobic exercise; $\mathrm{VO}_{2 \text { peak, }}$ peak oxygen uptake; 1-RM, 1-repetition maximum; $\mathrm{VO}_{2 \text { max }}$, maximum oxygen consumption; $\mathrm{OGT}$, oral glucose tolerance test; $\mathrm{LPA}$, leisure physical activity; HRR, heart rate reserve; $\mathrm{HR}_{\max }$, heart rate max; $\mathrm{Ml}$, motivational interview; NA, not available; NAFLD, nonalcoholic fatty liver disease; LP, linear periodization; DUP, daily undulating periodization.

the eight studies demonstrated significant improvements in insulin sensitivity following resistance exercise alone ${ }^{24,26}$ or combined with caloric restriction. ${ }^{33}$ Following 16 weeks of resistance exercise, Shaibi et al. ${ }^{24}$ demonstrated significant improvements in insulin sensitivity (45\%), as measured by frequently sampled intravenous glucose tolerance tests, compared with non-exercising controls $(-0.9 \%)$ in overweight Latino adolescent boys, and the changes in insulin sensitivity were independent of changes in total fat and lean body mass. Lee and colleagues also examined the effects of 3 months of resistance exercise versus aerobic exercise (without caloric restriction) on insulin sensitivity, as measured by a 3-hour hyperinsulinemiceuglycemic clamp technique, in previously sedentary adolescent boys $^{26}$ and girls ${ }^{32}$ with obesity. Although resistance exercise produced a significant improvement in insulin sensitivity (28\%) in adolescent boys, this was not the case in girls. Compared with the non-exercising controls, significant improvements in insulin sensitivity (33\%) were observed only following aerobic exercise in girls. These findings suggest the presence of sex differences in insulin sensitivity changes associated with resistance exercise training in adolescents. 
Currently, the effects of resistance exercise on oral glucose tolerance are not fully established, with only five randomized controlled trials completed to date. ${ }^{26,30,32-34}$ Only one of those studies ${ }^{33}$ demonstrated any significant improvements in 2-hour and insulin levels or glucose and insulin areas under the curve (AUCs) following 12-16 weeks of resistance exercise training with or without dietary modification. Suh et al. ${ }^{33}$ found that despite significant increases in body weight and visceral fat following a 12-week resistance exercise program with caloric restriction, insulin AUC was reduced from the baseline values; however those reductions did not differ significantly from the diet-only group in a mixed group of overweight Korean boys and girls.

\section{EFFECTS OF RESISTANCE EXERCISE ON TRADITIONAL CVD RISK FACTORS}

It is well known that obesity, particularly abdominal obesity, is associated with high blood pressure and dyslipidemia in children and adolescents, independent of BMI. ${ }^{10,11,55,56}$ Furthermore, adolescents with obesity have greater arterial stiffness and endothelial dysfunction than their lean counterparts. ${ }^{57-60}$ Aortic pulse wave velocity (aPWV), a measure of arterial stiffness, and carotid artery intima-media thickness (cIMT) have been suggested as markers of subclinical atherosclerosis and have been used as surrogate measures of cardiovascular events in adults. ${ }^{61}$ Iannuzzi et al. ${ }^{60}$ showed that both boys and girls with obesity (6-14 years) have significantly higher systolic and diastolic blood pressure and increased cIMT and arterial stiffness than their lean controls. Gungor et al. ${ }^{59}$ reported that aPWV is significantly increased in adolescents with obesity compared with their normal-weight peers. Increased cIMT and arterial stiffness in youth with obesity could increase their risk of atherosclerotic CVD if left untreated..$^{59,60}$

The effects of resistance exercise (alone or combined with a low caloric diet) on blood pressure and lipid profiles have been examined in children and adolescents with obesity (Table 3). ${ }^{31,35,36,62}$ Sung et al..$^{36}$ reported no reductions in total or low-density lipoprotein (LDL) cholesterol following 6 weeks of resistance exercise combined with a low caloric diet (900-1,200 kcal/day, 20\%-25\% fat) compared with the low caloric diet only group in Chinese children with obesity ( $8-11$ years, $>120 \%$ of the median weight for height). The previously mentioned 3-month intervention studies by Lee's group ${ }^{62}$ also found no significant changes in aPWV, cIMT, blood pressure, or lipid profiles following either resistance or aerobic exercise alone (e.g., no caloric restriction) despite significant reductions in body fat. Perhaps, the lack of improvements in traditional CVD markers could be due to a basement effect because the study participants had normal blood pressure and lipid values prior to the exercise intervention. Alternatively, these results could indicate that greater obesity reduction is required to improve CVD markers in youth with obesity. However, a recent meta-analysis of randomized controlled trials in adults ${ }^{63}$ reported that aerobic exercise, but not resistance exercise, was associated with significant improvements in arterial stiffness. Nevertheless, given the small number of randomized controlled trials and the relatively short resistance exercise interventions (8-16 weeks), the effects of resistance exercise alone on CVD markers are inconclusive in youth with obesity. Further studies with longer-term interventions are needed to verify the independent role of resistance exercise in improving traditional and non-traditional CVD markers in youth.

\section{EFFECTS OF RESISTANCE EXERCISE COMBINED WITH AEROBIC EXERCISE ON CARDIOMETABOLIC MARKERS}

In children and adolescents, several studies have examined whether combined exercise is better than aerobic exercise alone for improving total and abdominal fat (Table 1), insulin resistance (Table 2), and traditional CVD risk factors (Table 3). For example, Dâmaso et al. ${ }^{39}$ examined the effects of 12 months of combined aerobic and resistance exercise (3 times/wk, 60 minutes per session, $30 \mathrm{~min}$ utes of aerobic exercise at $50 \%-70 \%$ of $\mathrm{VO}_{2 \text { peak, }}$ and 30 minutes of resistance exercise at $6-20 \mathrm{RM} / 3$ sets) versus aerobic exercise alone ( 3 times/wk, 50 minutes per session, $50 \%-70 \%$ of $\mathrm{VO}_{2 \text { peak }}$ ) on total and abdominal fat and homeostatic model assessment for insulin resistance (HOMA-IR) in 139 adolescents with obesity (age 15-19 years). Although there were no significant differences in weight loss $(-12.3 \mathrm{~kg}$ vs. $-8.8 \mathrm{~kg})$ or HOMA-IR (-1.2 vs. -0.9$)$, reductions in total fat $(-14.2 \mathrm{~kg}$ vs. $-8.1 \mathrm{~kg})$, visceral fat $\left(-1.6 \mathrm{~cm}^{2}\right.$ vs. $\left.-1.4 \mathrm{~cm}^{2}\right)$, abdominal subcutaneous fat $\left(-0.9 \mathrm{~cm}^{2}\right.$ vs. $\left.-0.5 \mathrm{~cm}^{2}\right)$ and LDL cholesterol $(-12.1 \mathrm{mg} / \mathrm{dL}$ vs. $-4.8 \mathrm{mg} / \mathrm{dL})$ were all signifi- 


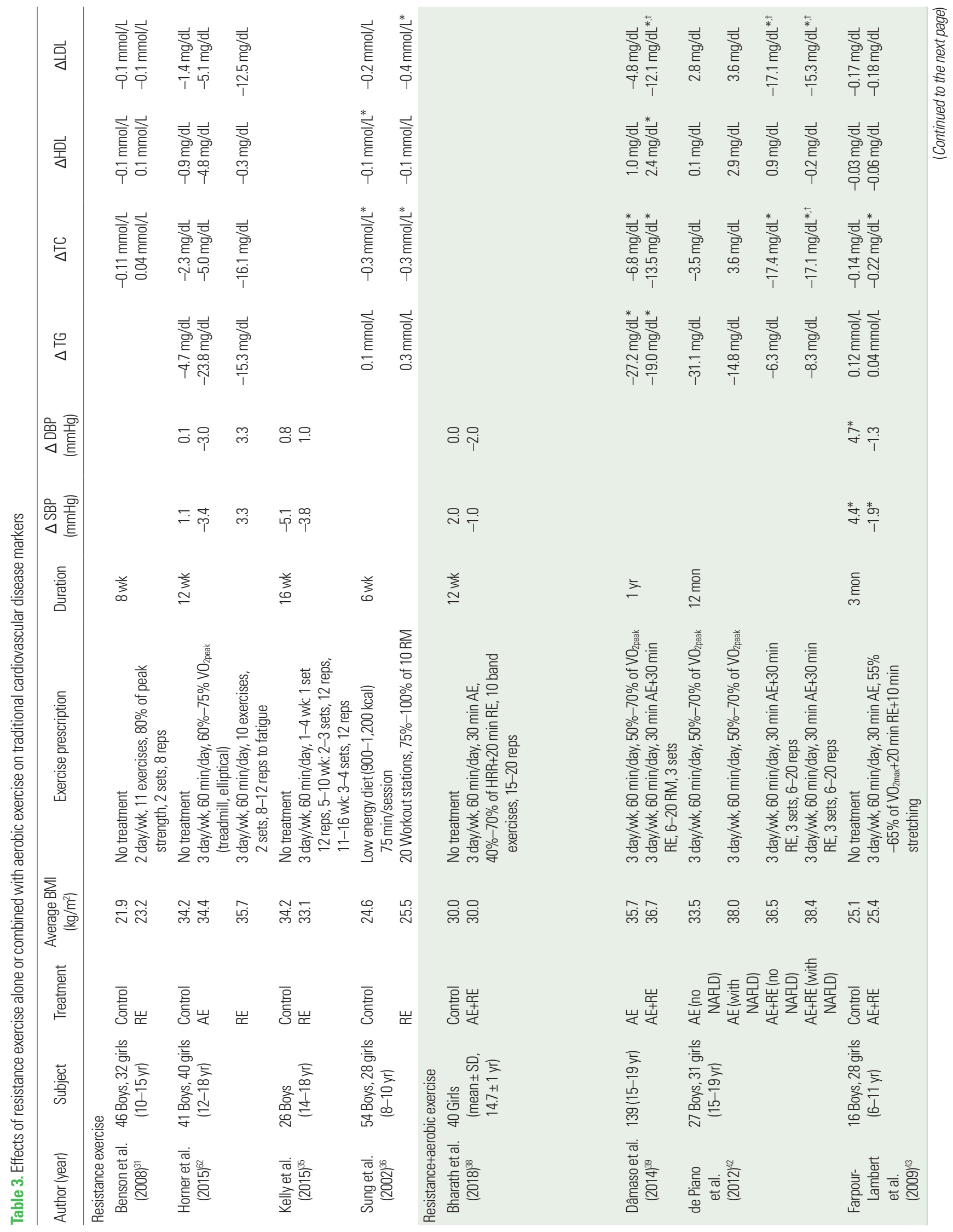




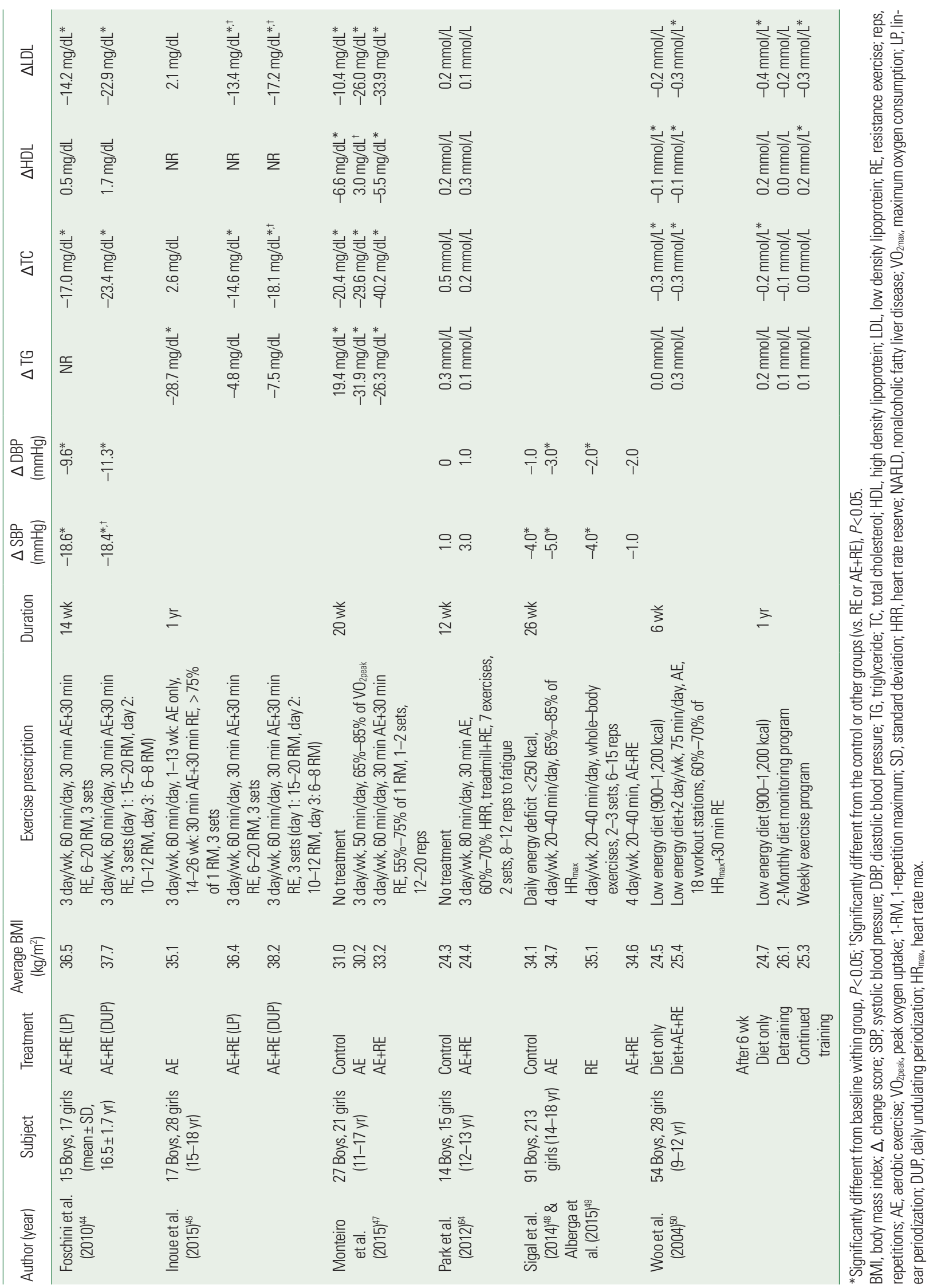


cantly greater in the combined exercise group than in the aerobic exercise only group. The same research group ${ }^{37}$ also examined the effects of 6 months of leisure-time physical activity, aerobic exercise alone, and combined aerobic and resistance exercise on body composition and insulin resistance in adolescents with obesity (age 1519 years). When the exercise time was matched between groups ( 3 times/wk, 60 minutes per session for 6 months), the reductions in body weight, BMI, and total fat mass and increases in FFM were significantly greater in the combined exercise group than in the aerobic exercise only group.

To the best of our knowledge, only two randomized trials ${ }^{46,48}$ have examined the effects of all three exercise modalities (aerobic, resistance, and combined exercise) together in a pediatric population. Sigal et al. ${ }^{48}$ investigated the influence of aerobic alone, resistance alone, and combined aerobic and resistance exercise training with caloric restriction (energy deficit $250 \mathrm{kcal} /$ day for all groups) on total body fat (\%) and cardiometabolic risk factors in a large sample of adolescents with obesity ( $\mathrm{n}=304$, age $14-18$ years). In that study ${ }^{48,49}$, all exercise groups had reduced total body fat (aerobic, $-1.2 \mathrm{~kg}$; resistance, $-1.3 \mathrm{~kg}$; combined, $-1.7 \mathrm{~kg}$ ) of a similar magnitude, as measured by the whole-body MRI technique. Abdominal subcutaneous fat also decreased similarly in all exercise groups (aerobic, $-16.2 \mathrm{~cm}^{2}$; resistance, $-22.7 \mathrm{~cm}^{2}$; combined exercise, $\left.-18.7 \mathrm{~cm}^{2}\right)$ compared with controls $\left(5.6 \mathrm{~cm}^{2}\right)$; however, no significant group differences were observed in the changes in visceral fat, fasting insulin, fasting glucose or 2-hour glucose, or lipid profiles. ${ }^{48,49}$

Recently, Lee et al. ${ }^{46}$ examined whether combined aerobic and resistance exercise is more effective than either aerobic or resistance exercise alone (without caloric restriction) in improving insulin sensitivity and reducing ectopic fat in the liver and skeletal muscle lipids in adolescents with overweight or obesity $(n=118$, age 12 17 years). In that study ${ }^{46}$, exercise duration was similar among groups (all groups performed 3 day/week, 60 minutes/session for 6 months), and the exercise compliance did not differ between groups (aerobic, 91\%; resistance, 89\%; and combined exercise, $89 \%)$. Although all three types of exercise reduced body fat (\%) and improved insulin sensitivity and oral glucose tolerance test 2-hour glucose, combined aerobic and resistance exercise and aerobic exercise alone were similarly beneficial in improving insulin sensitivity, and aerobic exercise alone was more effective than resistance exercise alone in improving insulin sensitivity.

\section{CONCLUSION}

Evidence demonstrates a strong association between muscular strength and cardiometabolic abnormalities in children and adolescents with obesity. Accordingly, current public physical activity guidelines ${ }^{22}$ recommend that children and adolescents engage in muscle strengthening physical activities at least 3 days a week, in addition to aerobic activity.

To date, only a few randomized trials have examined the effects of resistance exercise alone on total and abdominal obesity and cardiometabolic risk markers in children and adolescents with obesity. The pediatric literature contains wide variations in study design, resistance intervention duration (6 weeks-12 months) and frequency, subject characteristics (e.g., studying one or both sexes, pubertal status) and adherence to the prescribed exercise regimens. Limited evidence from well-designed randomized trials ${ }^{46,48}$ suggests that in the absence of weight loss, resistance exercise alone is associated with a significant increase in muscular strength and reductions in total fat and insulin resistance in previously sedentary adolescents with obesity. However, whether resistance training alone is associated with improvements in CVD factors is less clear. Nevertheless, incorporating resistance exercise into weight management interventions could be a useful strategy for improving muscular fitness and reducing obesity-related health risks in children and adolescents.

\section{CONFLICTS OF INTEREST}

The authors declare no conflict of interest.

\section{REFERENCES}

1. World Health Organization. Taking action on childhood obesity [Internet]. Geneva: World Health Organization; 2018 [cited 2019 Jun 21]. Available from: http://www.who.int/iris/handle/ $10665 / 274792$

2. Freedman DS, Khan LK, Serdula MK, Dietz WH, Srinivasan 
SR, Berenson GS. The relation of childhood BMI to adult adiposity: the Bogalusa Heart Study. Pediatrics 2005; 115:22-7.

3. Serdula MK, Ivery D, Coates RJ, Freedman DS, Williamson DF, Byers T. Do obese children become obese adults? A review of the literature. Prev Med 1993;22:167-77.

4. Sinha R, Fisch G, Teague B, Tamborlane WV, Banyas B, Allen $\mathrm{K}$, et al. Prevalence of impaired glucose tolerance among children and adolescents with marked obesity. N Engl J Med 2002; 346:802-10.

5. Cook S, Weitzman M, Auinger P, Nguyen M, Dietz WH. Prevalence of a metabolic syndrome phenotype in adolescents: findings from the third National Health and Nutrition Examination Survey, 1988-1994. Arch Pediatr Adolesc Med 2003;157:821-7.

6. Rocchini AP. Childhood obesity and a diabetes epidemic. $\mathrm{N}$ Engl J Med 2002;346:854-5.

7. Hannon TS, Rao G, Arslanian SA. Childhood obesity and type 2 diabetes mellitus. Pediatrics 2005;116:473-80.

8. Schwimmer JB, Deutsch R, Rauch JB, Behling C, Newbury R, Lavine JE. Obesity, insulin resistance, and other clinicopathological correlates of pediatric nonalcoholic fatty liver disease. J Pediatr 2003; 143:500-5.

9. Fernández JR, Bohan Brown M, López-Alarcón M, Dawson JA, Guo F, Redden DT, et al. Changes in pediatric waist circumference percentiles despite reported pediatric weight stabilization in the United States. Pediatr Obes 2017;12:347-55.

10. Janssen I, Katzmarzyk PT, Srinivasan SR, Chen W, Malina RM, Bouchard C, et al. Combined influence of body mass index and waist circumference on coronary artery disease risk factors among children and adolescents. Pediatrics 2005;115:1623-30.

11. Lee S, Bacha F, Arslanian SA. Waist circumference, blood pressure, and lipid components of the metabolic syndrome. J Pediatr 2006; 149:809-16.

12. Lee S, Bacha F, Gungor N, Arslanian SA. Waist circumference is an independent predictor of insulin resistance in black and white youths. J Pediatr 2006;148:188-94.

13. Lee S, Bacha F, Gungor N, Arslanian SA. Cardiorespiratory fitness in youth: relationship to insulin sensitivity and beta-cell function. Obesity (Silver Spring) 2006;14:1579-85.

14. Winsley RJ, Armstrong N, Middlebrooke AR, Ramos-Ibanez $\mathrm{N}$, Williams CA. Aerobic fitness and visceral adipose tissue in children. Acta Paediatr 2006;95:1435-8.

15. Christodoulos AD, Douda HT, Tokmakidis SP. Cardiorespiratory fitness, metabolic risk, and inflammation in children. Int J Pediatr 2012;2012:270515.

16. Lee S, Arslanian S. Body composition and cardiorespiratory fitness between metabolically healthy versus metabolically unhealthy obese black and white adolescents. J Adolesc Health 2019;64:327-32.

17. American College of Sports Medicine. ACSM's Guidelines for Exercise Testing and Prescription. Philadelphia (PA): Lippincott Williams \& Wilkins; 2018.

18. Ruiz JR, Sui X, Lobelo F, Lee DC, Morrow JR Jr, Jackson AW, et al. Muscular strength and adiposity as predictors of adulthood cancer mortality in men. Cancer Epidemiol Biomarkers Prev 2009; 18:1468-76.

19. Ruiz JR, Sui X, Lobelo F, Morrow JR Jr, Jackson AW, Sjöström $\mathrm{M}$, et al. Association between muscular strength and mortality in men: prospective cohort study. BMJ 2008;337:a439.

20. Benson AC, Torode ME, Singh MA. Muscular strength and cardiorespiratory fitness is associated with higher insulin sensitivity in children and adolescents. Int J Pediatr Obes 2006; 1: 222-31.

21. Artero EG, Lee DC, Lavie CJ, España-Romero V, Sui X, Church TS, et al. Effects of muscular strength on cardiovascular risk factors and prognosis. J Cardiopulm Rehabil Prev 2012;32: 351-8.

22. Piercy KL, Troiano RP, Ballard RM, Carlson SA, Fulton JE, Galuska DA, et al. The physical activity guidelines for Americans. JAMA 2018;320:2020-8.

23. Dietz WH. Childhood weight affects adult morbidity and mortality. J Nutr 1998;128(2 Suppl):411S-414S.

24. Shaibi GQ, Cruz ML, Ball GD, Weigensberg MJ, Salem GJ, Crespo NC, et al. Effects of resistance training on insulin sensitivity in overweight Latino adolescent males. Med Sci Sports Exerc 2006;38:1208-15.

25. Van Der Heijden GJ, Wang ZJ, Chu Z, Toffolo G, Manesso E, Sauer PJ, et al. Strength exercise improves muscle mass and hepatic insulin sensitivity in obese youth. Med Sci Sports Exerc 2010;42:1973-80.

26. Lee S, Bacha F, Hannon T, Kuk JL, Boesch C, Arslanian S. Ef- 
fects of aerobic versus resistance exercise without caloric restriction on abdominal fat, intrahepatic lipid, and insulin sensitivity in obese adolescent boys: a randomized, controlled trial. Diabetes 2012;61:2787-95.

27. Yu CC, Sung RY, Hau KT, Lam PK, Nelson EA, So RC. The effect of diet and strength training on obese children's physical self-concept. J Sports Med Phys Fitness 2008;48:76-82.

28. Goldfield GS, Kenny GP, Alberga AS, Prud'homme D, Hadjiyannakis S, Gougeon R, et al. Effects of aerobic training, resistance training, or both on psychological health in adolescents with obesity: The HEARTY randomized controlled trial. J Consult Clin Psychol 2015;83:1123-35.

29. Yu CC, Sung RY, So RC, Lui KC, Lau W, Lam PK, et al. Effects of strength training on body composition and bone mineral content in children who are obese. J Strength Cond Res 2005; 19:667-72.

30. Davis JN, Kelly LA, Lane CJ, Ventura EE, Byrd-Williams CE, Alexandar KA, et al. Randomized control trial to improve adiposity and insulin resistance in overweight Latino adolescents. Obesity (Silver Spring) 2009;17:1542-8.

31. Benson AC, Torode ME, Fiatarone Singh MA. The effect of high-intensity progressive resistance training on adiposity in children: a randomized controlled trial. Int J Obes (Lond) 2008;32:1016-27.

32. Lee S, Deldin AR, White D, Kim Y, Libman I, Rivera-Vega M, et al. Aerobic exercise but not resistance exercise reduces intrahepatic lipid content and visceral fat and improves insulin sensitivity in obese adolescent girls: a randomized controlled trial. Am J Physiol Endocrinol Metab 2013;305:E1222-9.

33. Suh S, Jeong IK, Kim MY, Kim YS, Shin S, Kim SS, et al. Effects of resistance training and aerobic exercise on insulin sensitivity in overweight Korean adolescents: a controlled randomized trial. Diabetes Metab J 2011;35:418-26.

34. Hasson RE, Adam TC, Davis JN, Kelly LA, Ventura EE, ByrdWilliams CE, et al. Randomized controlled trial to improve adiposity, inflammation, and insulin resistance in obese AfricanAmerican and Latino youth. Obesity (Silver Spring) 2012;20: 811-8.

35. Kelly LA, Loza A, Lin X, Schroeder ET, Hughes A, Kirk A, et al. The effect of a home-based strength training program on type 2 diabetes risk in obese Latino boys. J Pediatr Endocrinol Metab 2015;28:315-22.

36. Sung RY, Yu CW, Chang SK, Mo SW, Woo KS, Lam CW. Effects of dietary intervention and strength training on blood lipid level in obese children. Arch Dis Child 2002;86:407-10.

37. Ackel-D'Elia C, Carnier J, Bueno CR Jr, Campos RM, Sanches PL, Clemente AP, et al. Effects of different physical exercises on leptin concentration in obese adolescents. Int J Sports Med 2014;35:164-71.

38. Bharath LP, Choi WW, Cho JM, Skobodzinski AA, Wong A, Sweeney TE, et al. Combined resistance and aerobic exercise training reduces insulin resistance and central adiposity in adolescent girls who are obese: randomized clinical trial. Eur J Appl Physiol 2018;118:1653-60.

39. Dâmaso AR, da Silveira Campos RM, Caranti DA, de Piano A, Fisberg M, Foschini D, et al. Aerobic plus resistance training was more effective in improving the visceral adiposity, metabolic profile and inflammatory markers than aerobic training in obese adolescents. J Sports Sci 2014;32:1435-45.

40. Davis JN, Tung A, Chak SS, Ventura EE, Byrd-Williams CE, Alexander KE, et al. Aerobic and strength training reduces adiposity in overweight Latina adolescents. Med Sci Sports Exerc 2009;41:1494-503.

41.Davis JN, Gyllenhammer LE, Vanni AA, Meija M, Tung A, Schroeder ET, et al. Startup circuit training program reduces metabolic risk in Latino adolescents. Med Sci Sports Exerc 2011;43:2195-203.

42. de Piano A, de Mello MT, Sanches Pde L, da Silva PL, Campos RM, Carnier J, et al. Long-term effects of aerobic plus resistance training on the adipokines and neuropeptides in nonalcoholic fatty liver disease obese adolescents. Eur J Gastroenterol Hepatol 2012;24:1313-24.

43. Farpour-Lambert NJ, Aggoun Y, Marchand LM, Martin XE, Herrmann FR, Beghetti M. Physical activity reduces systemic blood pressure and improves early markers of atherosclerosis in pre-pubertal obese children. J Am Coll Cardiol 2009;54: 2396-406.

44. Foschini D, Araújo RC, Bacurau RF, De Piano A, De Almeida SS, Carnier J, et al. Treatment of obese adolescents: the influence of periodization models and ACE genotype. Obesity 
(Silver Spring) 2010;18:766-72.

45. Inoue DS, De Mello MT, Foschini D, Lira FS, De Piano Ganen A, Da Silveira, et al. Linear and undulating periodized strength plus aerobic training promote similar benefits and lead to improvement of insulin resistance on obese adolescents. J Diabetes Complications 2015;29:258-64.

46. Lee S, Libman I, Hughan K, Kuk JL, Jeong JH, Zhang D, et al. Effects of exercise modality on insulin resistance and ectopic fat in adolescents with overweight and obesity: a randomized clinical trial. J Pediatr 2019;206:91-8.

47. Monteiro PA, Chen KY, Lira FS, Saraiva BT, Antunes BM, Campos EZ, et al. Concurrent and aerobic exercise training promote similar benefits in body composition and metabolic profiles in obese adolescents. Lipids Health Dis 2015;14:153.

48. Sigal RJ, Alberga AS, Goldfield GS, Prud'homme D, Hadjiyannakis S, Gougeon R, et al. Effects of aerobic training, resistance training, or both on percentage body fat and cardiometabolic risk markers in obese adolescents: the healthy eating aerobic and resistance training in youth randomized clinical trial. JAMA Pediatr 2014;168:1006-14.

49. Alberga AS, Prud'homme D, Kenny GP, Goldfield GS, Hadjiyannakis S, Gougeon R, et al. Effects of aerobic and resistance training on abdominal fat, apolipoproteins and high-sensitivity C-reactive protein in adolescents with obesity: the HEARTY randomized clinical trial. Int J Obes (Lond) 2015;39:1494-500.

50. Woo KS, Chook P, Yu CW, Sung RY, Qiao M, Leung SS, et al. Effects of diet and exercise on obesity-related vascular dysfunction in children. Circulation 2004;109:1981-6.

51. Steinberger J, Daniels SR; American Heart Association Atherosclerosis, Hypertension, and Obesity in the Young Committee (Council on Cardiovascular Disease in the Young); American Heart Association Diabetes Committee (Council on Nutrition, Physical Activity, and Metabolism). Obesity, insulin resistance, diabetes, and cardiovascular risk in children: an American Heart Association scientific statement from the Atherosclerosis, Hypertension, and Obesity in the Young Committee (Council on Cardiovascular Disease in the Young) and the Diabetes Committee (Council on Nutrition, Physical Activity, and Metabolism). Circulation 2003;107:1448-53.

52. Hannon TS, Arslanian SA. The changing face of diabetes in youth: lessons learned from studies of type 2 diabetes. Ann N Y Acad Sci 2015;1353:113-37.

53. Zhang H, Zhang T, Li S, Li Y, Hussain A, Fernandez C, et al. Long-term impact of childhood adiposity on adult metabolic syndrome is modified by insulin resistance: the Bogalusa Heart Study. Sci Rep 2015;5:17885.

54. Faigenbaum AD, Kraemer WJ, Blimkie CJ, Jeffreys I, Micheli LJ, Nitka M, et al. Youth resistance training: updated position statement paper from the national strength and conditioning association. J Strength Cond Res 2009;23(5 Suppl):S60-79.

55. Savva SC, Tornaritis M, Savva ME, Kourides Y, Panagi A, Silikiotou $\mathrm{N}$, et al. Waist circumference and waist-to-height ratio are better predictors of cardiovascular disease risk factors in children than body mass index. Int J Obes Relat Metab Disord 2000;24:1453-8.

56. Freedman DS, Serdula MK, Srinivasan SR, Berenson GS. Relation of circumferences and skinfold thicknesses to lipid and insulin concentrations in children and adolescents: the Bogalusa Heart Study. Am J Clin Nutr 1999;69:308-17.

57. Tounian P, Aggoun Y, Dubern B, Varille V, Guy-Grand B, Sidi $D$, et al. Presence of increased stiffness of the common carotid artery and endothelial dysfunction in severely obese children: a prospective study. Lancet 2001;358:1400-4.

58. Cote AT, Phillips AA, Harris KC, Sandor GG, Panagiotopoulos C, Devlin AM. Obesity and arterial stiffness in children: systematic review and meta-analysis. Arterioscler Thromb Vasc Biol 2015;35:1038-44.

59. Gungor N, Thompson T, Sutton-Tyrrell K, Janosky J, Arslanian S. Early signs of cardiovascular disease in youth with obesity and type 2 diabetes. Diabetes Care 2005;28:1219-21.

60. Iannuzzi A, Licenziati MR, Acampora C, Salvatore V, Auriemma L, Romano ML, et al. Increased carotid intima-media thickness and stiffness in obese children. Diabetes Care 2004;27: 2506-8.

61. Salonen R, Salonen JT. Progression of carotid atherosclerosis and its determinants: a population-based ultrasonography study. Atherosclerosis 1990;81:33-40.

62. Horner K, Kuk JL, Barinas-Mitchell E, Drant S, DeGroff C, Lee $S$. Effect of aerobic versus resistance exercise on pulse wave velocity, intima media thickness and left ventricular mass in 
obese adolescents. Pediatr Exerc Sci 2015;27:494-502.

63. Ashor AW, Lara J, Siervo M, Celis-Morales C, Mathers JC. Effects of exercise modalities on arterial stiffness and wave reflection: a systematic review and meta-analysis of randomized controlled trials. PLoS One 2014;9:e110034.
64. Park JH, Miyashita M, Kwon YC, Park HT, Kim EH, Park JK, et al. A 12-week after-school physical activity programme improves endothelial cell function in overweight and obese children: a randomized controlled study. BMC Pediatr 2012;12: 111. 